\title{
A RETROSPECTIVE ANALYSIS OF FACIAL FRACTURE ETIOLOGIES
}

\author{
Martin Rubiev, Elitsa Deliverska \\ Department of Oral and Maxillofacial Surgery, Faculty of Dental Medicine, \\ Medical University - Sofia, Bulgaria
}

\section{ABSTRACT:}

Because of its close anatomic relations with nosal and oral cavitis, the maxillary sinus is the place of most frequent inflammatory diseases of all paranasal sinuses.

Introduction: The aim of this study was to establish differences in etiology and treatment of rhinogenic and odontogenic maxillary sinusitis.

Materials and methods: In this study, we analyzed the etiology, clinical characteristics of the disease, x-ray findings, clinical course and treatment of 188 cases, which were diagnosed and treated as odontogenic or rhinogenic maxillary sinusitis in the Departments of Maxillofacial surgery and Otorhinolaringology - "St. Anna" Hospital, Sofia from 2005 to 2010. Patients were divided according to age and sex. Data was systematized and analyzed.

Results: This study clearly showed that rhinogenic diseases of maxillary sinus are three times more frequent than odontogenic diseases. Also the etiology of odontogenic sinusitis most often is due to mistakes in the treatment of the upper teeth (alien corpuses, perforation after extraction), which is completely different from rhinogenic sinusitis. In the surgical treatment of rhinogenic maxillary sinusitis usually endonasal polypectomy was followed by operation according to Caldwell-Luc or functional sinus endoscopy. During the surgical treatment of odontogenic sinusitis the most frequent intervention was surgical plastic of oral-antral communication with mucogingival vestibular flap followed by operation according to Caldwell-Luck what is different from the treatment of rhinogenic sinusitis.

Conclusion: Maxillary sinus often suffers from inflammatory diseases and should always be examined carefully by means of anamnesis, clinic exam and x-rays to identify its origin. Upper teeth should be treated carefully in everyday's dental practice to avoid being cause of sinusitis.

Key words: Odontogenic Maxillary Sinusitis, Rhinogenic Maxillary Sinusitis

\section{PURPOSE:}

In the present study, we report our clinical investigations of cases of maxillary sinusitis caused by odontogenic and rhinogenic factors in order to establish the similarities and the differences in theirs etiology, diagnosis and treatment.

\section{METHODS:}

In our study we used the data gathered from the medical records of 188 patients whit maxillary sinusitis that underwent treatment (both surgical and conservative) in our clinic for the period of June 2005 to February 2010. Cases were divided according to several factors (diagnosis, etiology, underwent treatment, age, gender, etc.).

\section{RESULTS:}

Most of the patients treated in our clinic with maxillary sinusitis where directed to us either by their general practitioner or by their dentist. Very few of them sought medical help directly into the clinic.

The common complaints for the odontogenic cases were oral symptoms such as swelling, pain and discomfort, and the formation of oroantral communication or fistula into maxillary sinus. The most common causative tooth was the first molar, followed by the second molar and the second bicuspid. We classified the causative factors of the disease into apical lesion; post extractive oro-antral communication or fistula; intra-sinus foreign body; ectopic teeth in the maxillary sinus; odontogenic cysts disrupting Schneidarian membrane and placement of dental implants. The classification showed that the spread of inflammation due to apical lesion into maxillary sinus was the most common cause. Iatrogenic causes such as dental extractions and forming oro-antral fistula were also common.

There was no significant difference in the morbidity between males and females in both odontogenic and rhinogenic sinusitis, neither between involvement of the left or right maxillary sinus. However it should be mentioned that the rhinogenic cases were more often acute and also involved other paranasal cavities in the inflammatory process, such as ethmoidal cells, frontal sinus etc. The common x-ray findings in the Waters' projection (falling of the bottom of maxillary sinus and the cloudy shadow of maxillary sinus) showed no difference between sinusitis of odontogenic and rhinogenic source except in cases caused by impacted teeth or cysts growing into the sinus cavity. 
Most of the patients with sinusitis of rhinogenic origin were treated with conservative therapy (antibiotics, endonasal washing by punction). All cases of odontogenic source needed some surgical treatment (classical CaldwellLuc, Rehrmann's buccal flap to close the oro-antral fistula or just extraction of the causative tooth).

$\begin{array}{lcc}\text { Acute sinusitis } & 78 & 11 \\ \text { Chronic sinusitis } & 57 & 42 \\ \text { Conservative treatment } & 84 & 0 \\ \text { Surgical treatment } & 51 & 53 \\ \text { Male } & 61 & 27 \\ \text { Female } & 74 & 26 \\ \text { Age up to } 21 & 56 & 5 \\ \text { Age between } 22 \text { and } 60 & 61 & 44 \\ \text { Age 61 and more } & 18 & 4\end{array}$

\section{CONCLUSION:}

The association between an odontogenic and rhinogenic condition and maxillary sinusitis requires a thorough dental, rhino and x-ray examination of patients with sinusitis. Concomitant management of the dental origin and the associated sinusitis will ensure complete resolution of the infection and may prevent recurrences and complications. A combination of a medical and surgical approach is generally required for the treatment of odontogenic sinusitis.
Fig. 1: Gender

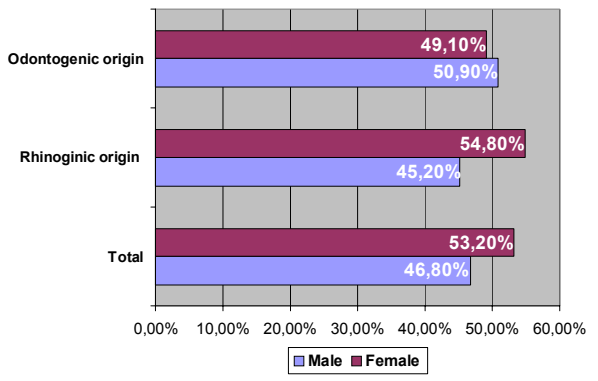

Fig. 2: Age

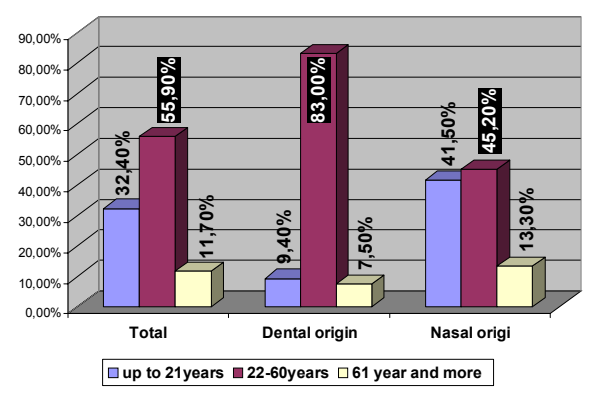

Fig. 3: Etiology and clinical course

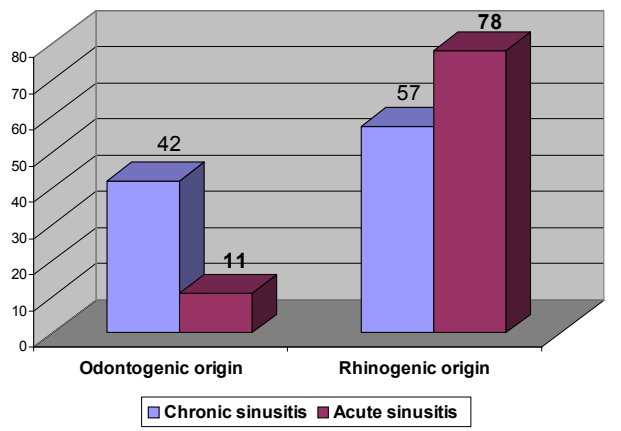

\section{REFERENCES:}

1. Albu S. Tomescu E. Small and large middle meatus antrostomies in the treatment of chronic maxillary sinusitis. Otolaryngol Head Neck Surg. 2004 Oct; 131(4):542-547. [PubMed] [CrossRef]

2. Baykul T. Dopru H. Yasan H. Cina Aksoy M. Clinical impact of ectopic teeth in the maxillary sinus. Auris Nasus Larynx. 2006 Sep; 33(3):277-281 [PubMed] [CrossRef]

3. Costa F. Emanuelli E. Robiony M. Zerman N. Polini F. Politi M. Endoscopic Surgical Treatment of Chronic Maxillary Sinusitis of Dental Origin. J Oral Maxillofac Surg. 2007
Feb;65(2):223-8. [PubMed] [CrossRef] 4. Hunter WL 4th. Bradrick JP. Houser SM. Patel JB. Sawady J. Maxillary sinusitis resulting from ostium plugging by dislodged bone graft: case report. J Oral Maxillofac Surg. 2009 Jul;67(7):1495-1498. [PubMed]

\footnotetext{
Address for correspondence:

Martin Rubiev

Department of Oral and Maxillofacial surgery, Faculty of Dental Medicine, Medical University, Sofia

1, Georgi Sofiiski Str., 1431 Sofia, Bulgaria

E-mail:mrubiev@yahoo.com
} 